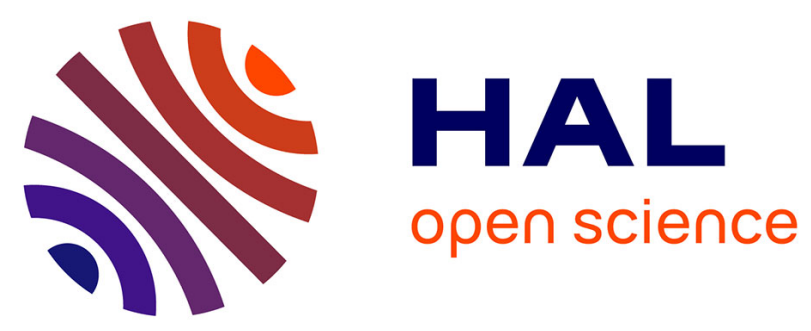

\title{
Molecules probed with a slow chirped-pulse excitation: Analytical model of the free-induction-decay signal
}

\author{
C. Bray, Gaël Mouret, A. Cuisset, Francis Hindle, Robin Bocquet, Kevin \\ Hickson, Guillaume Dhont, Daniele Fontanari
}

\section{- To cite this version:}

C. Bray, Gaël Mouret, A. Cuisset, Francis Hindle, Robin Bocquet, et al.. Molecules probed with a slow chirped-pulse excitation: Analytical model of the free-induction-decay signal. Physical Review A, 2019, 100 (4), pp.043407. 10.1103/PhysRevA.100.043407 . hal-02322177

\section{HAL Id: hal-02322177 \\ https://hal.science/hal-02322177}

Submitted on 17 Nov 2020

HAL is a multi-disciplinary open access archive for the deposit and dissemination of scientific research documents, whether they are published or not. The documents may come from teaching and research institutions in France or abroad, or from public or private research centers.
L'archive ouverte pluridisciplinaire HAL, est destinée au dépôt et à la diffusion de documents scientifiques de niveau recherche, publiés ou non, émanant des établissements d'enseignement et de recherche français ou étrangers, des laboratoires publics ou privés. 


\title{
Molecules probed with a slow chirped pulse excitation: analytical model of the free induction decay signal
}

\author{
D. Fontanari, C. Bray, G. Dhont, G. Mouret, A. Cuisset, F. Hindle, and R. Bocquet* \\ LPCA, Université du Littoral Côte d'Opale, 189A avenue Maurice Schumann, 59140 Dunkerque, France \\ K. M. Hickson \\ ISM, Université de Bordeaux, 351 cours de la libération, 33405 Talence cedex, France
}

(Dated: August 29, 2019)

\begin{abstract}
Most of chirped pulse experiments refer to a theoretical study from [J. C. McGurk, T. G. Schmalz, and W. H. Flygare, J. Chem. Phys. 60, 4181 (1974)] which is well tailored to interpret the signals obtained with very fast chirped pulses, but is not sufficient to account for the signals in the case of slower chirped pulses used in spectroscopy to increase the signal to noise ratio. A theoretical study of the polarization of molecules in a cell, uniform supersonic jet or molecular beam and subjected to a chirped pulse is presented. Three degrees of approximation for the polarization are introduced and are compared with the numerical solution of the optical Bloch equations. These expressions enter the analytic expression of the free induction decay signal which is validated against experimental data on the rotational emission spectra of OCS molecules. A relation between the pulse duration, the line position in the chirped pulse and the signal amplitude is proposed in the thermalized case. It assists in the optimization of the chirped pulse parameters and in the estimation of the error associated with the line intensity.
\end{abstract}

\section{INTRODUCTION}

Chirped Pulse (CP) spectrometers are now widely employed at microwave and millimeter frequencies to perform high resolution spectroscopic studies [1-5]. A small number of instruments have also been set up in the submillimeter band [6]. Such systems employ a two-step measurement cycle. Firstly, the gas being studied is polarized by a CP emitted from a powerful source. Secondly, after the source extinction, the emission of the molecules corresponding to the Free Induction Decay (FID) is recorded. The signal level is proportional to the polarization reached at the end of the CP. The spectrum of the molecular emission is then recovered by the use of a Fourier transform. The broadband nature of CP spectroscopy has the advantage that many rotational transitions may be probed simultaneously.

While this technique is very well suited to the frequency measurement of rotational transitions, measurements of line strengths require more care. It has already been shown that the position of the rotational transition frequency in the pulse can affect the measured intensity, especially in cases where pulse durations are increased to obtain sufficient signal to noise ratios [5, 7]. However, most groups use a model originally developed by McGurk et al. [8] to model FID signals. It neglects re-emission effects during the pulse, together with population and coherence relaxation times, due to a very fast passage on the transition. In the model, the polarization at the end of the pulse is proportional to $\sqrt{T_{c} / \Delta \omega}$ where $T_{c}$ is the pulse duration and $\Delta \omega$ is the spectral extension of the CP. This behaviour is in contradiction with the experi-

\footnotetext{
*robin.bocquet@univ-littoral.fr
}

mental measurements for which we observe a maximum FID signal for a particular pulse duration [5].

This work proposes new analytical expressions for the polarization which will be helpful to analyze the experiments with slower CP. Section II deals with the polarizing step where the molecules evolve under the influence of the CP. We propose three approximations and compare them with the numerical integration of the optical Bloch equations. Both thermalized cases (cell and uniform supersonic jet) and non-thermalized cases (molecular beam) are discussed. Section III presents the second step of a CP experiment, i.e. the Free Induction Decay. Results of Section II are leveraged to propose an analytical expression of the FID recorded signal. This formula is validated through comparison with experimental data in the thermalized case using an experimental set-up described in [5], similar to those of $[2,4,6,9]$. We show how to use this expression to optimize the amplitude of the signal and to take into account the dependence of the intensity on the line position.

\section{ANALYTICAL EXPRESSION OF THE POLARIZATION IN A CP EXPERIMENT}

\section{A. Optical Bloch equations}

The molecular interaction with an electromagnetic wave has been extensively described in the literature [8, 10-13]. We discuss for simplicity a two-level isolated molecular system, given by its energy levels $E_{a}$ and $E_{b}$ with $E_{a}-E_{b}=\hbar \omega_{0}$. We consider the interaction between this two-level system and an electromagnetic field with angular frequency $\omega(t)$. The system of $N$ molecules with a transition dipole moment $\mu_{a b}$ is described by the evolution of its density matrix. Introducing the relaxation 
mechanisms by $\gamma_{1}=1 / T_{1}$ and $\gamma_{2}=1 / T_{2}$ (respectively, inverse of the decay time of the population difference and inverse of the dipole dephasing time) and $W_{\text {eq }}$, the population difference at thermodynamic equilibrium, we get the generic form of the optical Bloch equations:

$$
\begin{aligned}
\frac{d z}{d t}(t) & =-\left(\gamma_{2}+i \omega_{0}\right) z(t)-i \mathcal{E}(t) W(t), \\
\frac{d W}{d t}(t) & =-\gamma_{1}\left(W(t)-W_{\mathrm{eq}}\right)+\frac{1}{2 i}\left(\mathcal{E}^{*}(t) z(t)-\mathcal{E}(t) z^{*}(t)\right),
\end{aligned}
$$

where $W(t)$ and $z(t)=\mathscr{P}(t) /\left(N \mu_{a b}\right)$ correspond to the difference of population and the pseudo-polarization respectively, $\mathscr{P}(t)$ being the polarization. The function $\mathcal{E}(t)=e^{-i \alpha(t)} \Omega_{0}$ represents the perturbation of the system with $\Omega_{0}=\frac{\mu_{a b} E_{0}}{\hbar}$ the Rabi frequency and $\alpha(t)$ a generic function of time. Equations (1) and (2) are solved for given functions $W(t)$ and $z(t)$ respectively. Using the general solution of a first order differential equation, we have:

$$
\begin{aligned}
z(t)= & z(0) e^{-\left(i \omega_{0}+\gamma_{2}\right) t}-i \int_{0}^{t} e^{-\left(i \omega_{0}+\gamma_{2}\right)(t-x)} \mathcal{E}(x) W(x) \mathrm{d}(\not{x} .) \\
W(t)= & W_{\mathrm{eq}}+\left(W(0)-W_{\mathrm{eq}}\right) e^{-\gamma_{1} t}+ \\
& \frac{1}{2 i} \int_{0}^{t} e^{-\gamma_{1}(t-x)}\left(\mathcal{E}^{*}(x) z(x)-\mathcal{E}(x) z^{*}(x)\right) \mathrm{d} x .
\end{aligned}
$$

We suppose that $z(t)$ and $W(t)$ may be written as series:

$$
\begin{aligned}
z(t) & =z^{(0)}(t)+z^{(1)}(t)+z^{(2)}(t)+\cdots \\
W(t) & =W^{(0)}(t)+W^{(1)}(t)+W^{(2)}(t)+\cdots
\end{aligned}
$$

Replacing these series in the Eq. (3) and Eq.(4) and identifying term by term, we find the series recurrence:

$$
\begin{aligned}
z^{(n+1)}(t) & =-i \int_{0}^{t} e^{-\left(i \omega_{0}+\gamma_{2}\right)(t-x)} \mathcal{E}(x) W^{(n)}(x) \mathrm{d} x \\
W^{(n+1)}(t) & =\frac{1}{2 i} \int_{0}^{t} e^{-\gamma_{1}(t-x)}\left(\mathcal{E}^{*}(x) z^{(n)}(x)-\mathcal{E}(x) z^{(n) *}(x)\right) \mathrm{d} x .
\end{aligned}
$$

\section{B. Mathematical formulation}

We consider in the paper a linear $\mathrm{CP}$ with duration $T_{c}$ and spectral extension $\Delta \omega$. The CP frequency equals the molecular resonance at time $r_{0} T_{c}$ with $r_{0} \in[0 ; 1]$, the relative line position in the $\mathrm{CP}$ (for $r_{0}=\frac{1}{2}$ the chirp is centered on the resonance $\left.\omega_{0}\right)$. The angular frequency is given by:

$$
\omega(t)=\frac{d \alpha}{d t}(t)=\omega_{0}-r_{0} \Delta \omega+\frac{\Delta \omega}{T_{c}} t .
$$

Except for free induction decay or double resonance experiments, we are dealing with $z(0)=0$. Only the odd terms of the $z$-series and the even terms of the $W$-series are non-zero. In particular, the first non zero terms of the series are:

$$
\begin{aligned}
z^{(1)}(t) & =-i \int_{0}^{t} e^{-\left(i \omega_{0}+\gamma_{2}\right)(t-x)} \mathcal{E}(x) W^{(0)}(x) \mathrm{d} x, \\
W^{(0)}(t) & =W_{\text {eq }}+\left(W(0)-W_{\text {eq }}\right) e^{-\gamma_{1} t} .
\end{aligned}
$$

A rotating wave approximation is performed by introducing $\xi^{(n)}(t)=e^{i \alpha(t)} z^{(n)}(t)$. Defining $\beta^{2}=\frac{\Delta \omega}{T_{c}}$, the sweep speed of the pulse, the pseudo-polarization is then given by:

$$
\xi^{(1)}(t)=W_{\text {eq }} g\left(t, \gamma_{2}\right)+\left(W(0)-W_{\text {eq }}\right) e^{-\gamma_{1} t} g\left(t, \gamma_{2}-\gamma_{1}\right),
$$

where

$$
g(t, \gamma)=-i \Omega_{0} \int_{0}^{t} e^{-\gamma(t-x)-i c \beta^{2}(t-x) T_{c}+i \frac{\beta^{2}}{2}\left(t^{2}-x^{2}\right)} \mathrm{d} x
$$

which can be integrated using the error function:

$$
g(t, \gamma)=\rho(t, \gamma) e^{i \theta(t, \gamma)} \sigma(t, \gamma)
$$

where

$$
\begin{aligned}
\rho(t, \gamma)= & \frac{\sqrt{\pi} \Omega_{0}}{\sqrt{2} \beta} \exp \left(-\gamma\left(t-r_{0} T_{c}\right)\right) \\
\theta(t, \gamma)= & \left(t-r_{0} T_{c}\right)^{2} \frac{\beta^{2}}{2}-\frac{3}{4} \pi-\frac{\gamma^{2}}{2 \beta^{2}} \\
\sigma(t, \gamma)= & \operatorname{erf}\left(\frac{e^{i \frac{\pi}{4}}}{\sqrt{2}}\left(r_{0} T_{c} \beta-i \frac{\gamma}{\beta}\right)\right)+ \\
& \operatorname{erf}\left(\frac{e^{i \frac{\pi}{4}}}{\sqrt{2}}\left[\left(t-r_{0} T_{c}\right) \beta+i \frac{\gamma}{\beta}\right]\right) .
\end{aligned}
$$

At time $t=T_{c}$ (polarization at the end of the pulse), we can simplify the expressions by ignoring small terms $\left(\frac{\gamma}{\beta} \ll 1\right)$ and taking a first order asymptotic expansion of the error function [14] in order to obtain:

$$
g_{\text {asy }}\left(T_{c}, \gamma\right)=\rho_{\text {asy }}\left(T_{c}, \gamma\right) e^{i \theta_{\text {asy }}\left(T_{c}\right)}+g_{\text {osc }}\left(T_{c}, \gamma\right),
$$

where

$$
\begin{aligned}
\rho_{\text {asy }}\left(T_{c}, \gamma\right)= & \frac{\sqrt{2 \pi} \Omega_{0}}{\sqrt{\Delta \omega}} \sqrt{T_{c}} \exp \left[-T_{c}\left(1-r_{0}\right) \gamma\right], \\
\theta_{\text {asy }}\left(T_{c}\right)= & \left(1-r_{0}\right)^{2} \frac{\Delta \omega}{2} T_{c}-\frac{3}{4} \pi, \\
g_{\text {osc }}\left(T_{c}, \gamma\right)= & \frac{\Omega_{0}}{\Delta \omega}\left(\frac{1}{1-r_{0}}+\frac{1}{r_{0}} \exp \left[i \frac{\Delta \omega}{2} T_{c}\left(1-2 r_{0}\right)\right]\right) \times \\
& \exp \left[-T_{c}\left(1-r_{0}\right) \gamma\right] .
\end{aligned}
$$

Using Eq. (9), $\xi^{(1)}\left(T_{c}\right)$ is then approximated by:

$$
\begin{aligned}
\xi_{\text {asy }}^{(1)}\left(T_{c}\right)= & W_{\text {eq }} g_{\text {asy }}\left(T_{c}, \gamma_{2}\right) \\
& +\left(W(0)-W_{\text {eq }}\right) e^{-\gamma_{1} T_{c}} g_{\text {asy }}\left(T_{c}, \gamma_{2}-\gamma_{1}\right) .
\end{aligned}
$$


The term $g_{\text {osc }}\left(T_{c}, \gamma\right)$ induces an oscillation whose amplitude decreases as $T_{c}$ and/or $\Delta \omega$ increases. We neglect it to get an approximation of the pseudo-polarization:

$$
\begin{aligned}
\xi_{\text {app }}^{(1)}\left(T_{c}\right)= & e^{i \theta_{\text {asy }}\left(T_{c}\right)}\left[W_{\text {eq }} \rho_{\text {asy }}\left(T_{c}, \gamma_{2}\right)\right. \\
& \left.+\left(W(0)-W_{\text {eq }}\right) e^{-\gamma_{1} T_{c}} \rho_{\text {asy }}\left(T_{c}, \gamma_{2}-\gamma_{1}\right)\right] .
\end{aligned}
$$

Finally, the modulus of the pseudo-polarization is approximated by $\left|\xi\left(T_{c}\right)\right| \approx\left|\xi^{(1)}\left(T_{c}\right)\right| \approx\left|\xi_{\text {asy }}^{(1)}\left(T_{c}\right)\right| \approx$ $\left|\xi_{\text {app }}^{(1)}\left(T_{c}\right)\right|$, with

$$
\begin{aligned}
\left|\xi_{\text {app }}^{(1)}\left(T_{c}\right)\right|= & \sqrt{2 \pi} \Omega_{0} \sqrt{\frac{T_{c}}{\Delta \omega}} \times e^{-\frac{T_{c}}{T_{2}}\left(1-r_{0}\right)} \\
& \times\left|W_{\text {eq }}+\left(W(0)-W_{\text {eq }}\right) e^{-\frac{T_{c}}{T_{1}} r_{0}}\right|,
\end{aligned}
$$

which is the product of three terms. The first one corresponds to the polarization in the McGurk approximation [8]. The second one describes an exponential decay with relaxation time $T_{2}$, for a duration of $T_{c}\left(1-r_{0}\right)$ (duration between the line position and the end of the pulse). The third term represents the relaxation of the difference population towards the equilibrium with a relaxation time $T_{1}$, for a duration $r_{0} T_{c}$ (duration between the beginning of the pulse and the line position).

\section{Numerical validation of the approximations}

Figure 1 compares the three approximations $\left(\left|\xi^{(1)}\left(T_{c}\right)\right|, \quad\left|\xi_{\text {asy }}^{(1)}\left(T_{c}\right)\right|, \quad\left|\xi_{\text {app }}^{(1)}\left(T_{c}\right)\right|\right)$ of the modulus of the polarization with the numerical integration of the optical Bloch equations using a Rosenbrock method [15] for three different spectral extensions and three different line positions $r_{0}$. We consider a two-level system consisting of the $J=16$ and $J=17$ rotational levels of the OCS molecule with $W(0)=W_{\text {eq }}$ and $T_{2}=T_{1}=0.3 \mu \mathrm{s}$. We notice that except for the case $\Delta \nu=10 \mathrm{MHz}$ and $r_{0}=0.9$ of Fig. 1(c), the first approximation $\left|\xi^{(1)}\left(T_{c}\right)\right|$ given by Eq. (9) (green curve) reproduces the numerical integration (blue curve). As soon as $\Delta \nu \geqslant 100 \mathrm{MHz}$, the red curves corresponding to the second approximation $\left|\xi_{\text {asy }}^{(1)}\left(T_{c}\right)\right|($ Eq. (15)) are acceptable. The black curves corresponding to the third approximation $\left|\xi_{\text {app }}^{(1)}\left(T_{c}\right)\right|$ (Eq. (16)) have no more oscillations and reproduce the global behavior of the numerical results, in particular the optimal pulse duration and the corresponding value of the polarization. Figure 1 suggests however two remarks. First, the asymptote of the modulus of the polarization for high $T_{c}$ value is not zero. This effect is seen for $r_{0} \leqslant 0.5$ and would be visible at $T_{c}>10 \mu$ s for $r_{0}=0.9$. This asymptote decreases when $\Delta \nu$ increases and $/$ or $r_{0}$ decreases. Second, for $\Delta \nu=1000 \mathrm{MHz}$, the amplitude of the oscillations decreases from $r_{0}=0.1$ to $r_{0}=0.5$ but increases from $r_{0}=0.5$ to $r_{0}=0.9$.

\section{Discussion}

The molecules interacting with a chirped pulse can be probed under different experimental conditions: in an ordinary cell [5, 16, 17], in a uniform supersonic jet [7], or in a molecular beam $[2,3,18]$. We can take advantage of the typical characteristics of each experimental setup summed up in Table I to adapt the analytic approximation (17) of the modulus of the polarization obtained in Section II B.

In an ordinary cell or in a uniform supersonic jet, the molecules are thermalized. The rotational temperature may be the ambient temperature in the former situation while it is lowered to a few dozens of kelvins in the latter one. In both cases, the relaxation time $T_{2}$ is of the same order of magnitude as $T_{1}$ and the initial population difference $W(0)$ corresponds to its equilibrium value $W_{\text {eq }}$. In these conditions, the analytic approximation of Eq. (17) is proportional to $\left|W_{\text {eq }}\left(T_{\text {rot }}\right)\right|$ which can be significantly increased in a uniform supersonic jet with respect to an ordinary cell at $300 \mathrm{~K}$ by decreasing the temperature. The pulse duration giving the maximal polarization is found to be $T_{c, \max }=T_{2} /\left(2\left(1-r_{0}\right)\right)$, which depends on $T_{2}$ but not on $T_{1}$.

The behaviour of experiments in the non-thermalized case is different. In a molecular beam experiment, the rotational temperature is lowered to a few kelvins, the difference population $W(0)$ can be as high as $50 W_{\text {eq }}$. The analytic approximation of Eq. (17) simplifies to:

$$
\left|\xi_{\text {app }}^{(1)}\left(T_{c}\right)\right| \propto \sqrt{\frac{T_{c}}{\Delta \omega}} e^{-\frac{T_{c}}{T_{2}}\left(1-r_{0}\right)}|W(0)| e^{-\frac{T_{c}}{T_{1}} r_{0}} .
$$

The collisional linewidth is strongly reduced, implying generally $T_{2}, T_{1} \gg T_{c}[13,19]$. Under these conditions, the modulus of polarization reduces to the McGurk's model [8]. The optimal pulse duration is then no longer visible. The molecular beam chirped pulse setup is very advantageous in term of signal to noise ratio. First, the signal is enhanced by the difference populations $W(0)$. Second, the duration of the FID signal increases due to the relaxation time $T_{2}$ (see III) to such an extent that the decay of the FID signal is often driven by the Doppler inhomogeneous broadening (see Appendix A). The Doppler width is indeed proportional to $\omega_{0}$ (see Eq. (A5)) and more pronounced in the millimeter or submillimeter ranges than in the microwave range. Unfortunately, the drawback of a molecular beam experiment is a limited operating frequency range due to the peaked shape of the population of energy levels towards the low $J$ values [20].

\section{FREE INDUCTION DECAY SIGNAL}

\section{A. Mathematical formulation}

The source extinction occurs at $t=T_{c}$. The emission of the polarized molecules which evolve freely is observed 

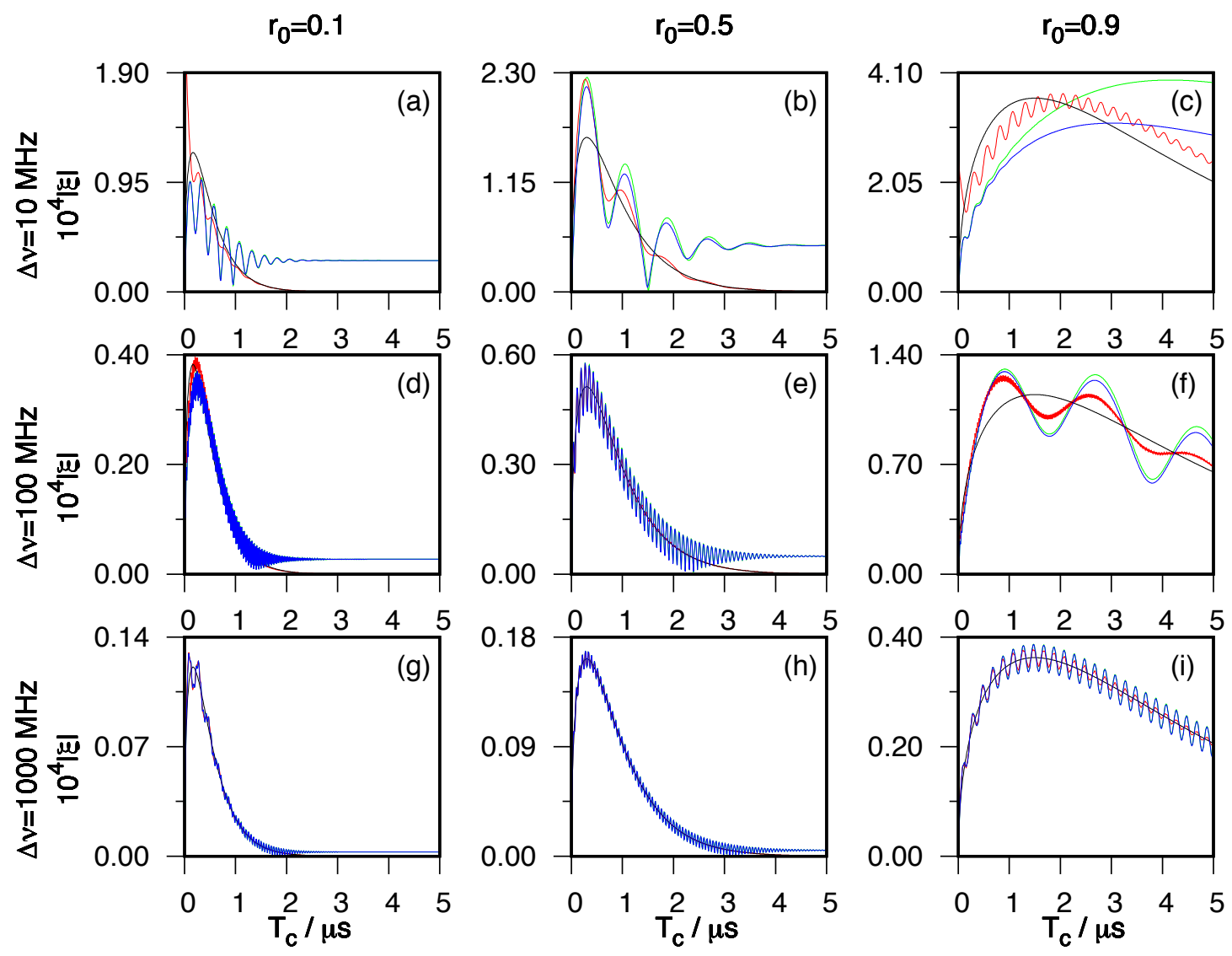

Figure 1: Polarization modulus at the end of the $\mathrm{CP}$ against the chirp duration $T_{c}$ for three spectral extensions $\Delta \nu \in\{10,100,1000\} \mathrm{MHz}$ and three line positions $r_{0} \in\{0.1,0.5,0.9\}$. Blue curves: numerical integration of optical

Bloch equations. Green curves: first non zero term $\left|\xi^{(1)}\left(T_{c}\right)\right|$ of the series. Red curves: asymptotic expansion $\left|\xi_{\text {asy }}^{(1)}\left(T_{c}\right)\right|$ of the polarization. Black curves: polarization $\left|\xi_{\text {app }}^{(1)}\left(T_{c}\right)\right|$ after the last approximation. Parameters are: $J=17 \rightarrow 16$ rotational transition of OCS at $206.745 \mathrm{GHz}, \Omega_{0}=2.4 \mathrm{rd} / \mu \mathrm{s}, W(0)=W_{\text {eq }}=610^{-4}, T_{2}=T_{1}=0,3 \mu \mathrm{s}$.

with time $t^{\prime}=t-T_{c}$. The polarization in the rotating wave approximation $\tilde{\mathscr{P}}\left(t^{\prime}\right)$ is given by (see Appendix A):

$$
\tilde{\mathscr{P}}\left(t^{\prime}\right)=N \mu_{a b} \xi\left(T_{c}\right) \exp \left[-\frac{t^{\prime}}{T_{2}}-\frac{t^{\prime 2} \Delta \omega_{D}^{2}}{4 \ln 2}\right]
$$

where $\Delta \omega_{D}$ is the Doppler width. The electric field is emitted at angular frequency $\omega_{0}$, the molecular resonance, with an amplitude proportional to the polarization module [2]:

$$
E\left(t^{\prime}\right) \propto\left|\tilde{\mathscr{P}}\left(t^{\prime}\right)\right| e^{-i\left(\omega_{0} t^{\prime}+\Phi\right)}+c c
$$

Chirped pulse experiments use an heterodyne detection scheme [5] where a local oscillator of angular frequency $\omega_{L O}$ is used to shift the spectrum from $\omega_{0}$ to the intermediate angular frequency $\omega_{I F}=\omega_{0}-\omega_{L O}$. If $\phi$ is a phase term at the time origin, the output signal is (see Appendix B):

$$
\begin{aligned}
S\left(t^{\prime}\right) \propto & N \mu_{a b}\left|\xi_{\text {app }}^{(1)}\left(T_{c}\right)\right| \\
& \times e^{-\frac{t^{\prime}}{T_{2}}} e^{-\frac{t^{\prime 2} \Delta \omega_{D}^{2}}{4 \ln 2}} \times \cos \left(\omega_{I F} t^{\prime}+\phi\right) .
\end{aligned}
$$

The exponential terms describe dampings respectively due to the dephasing polarization and Doppler broadening. The last term is an oscillation at the intermediate angular frequency $\omega_{I F}$.

\section{B. Experimental results}

$\mathrm{CP}$ experiments have been performed in order to check the validity of the FID signal model of Eq. (20) in the case of a thermalized sample $\left(W(0)=W_{\text {eq }}\right)$. We recorded the FID signal of the OCS molecule around $206.745 \mathrm{GHz}$ corresponding to the rotational transition $J=17 \rightarrow 16$. For 


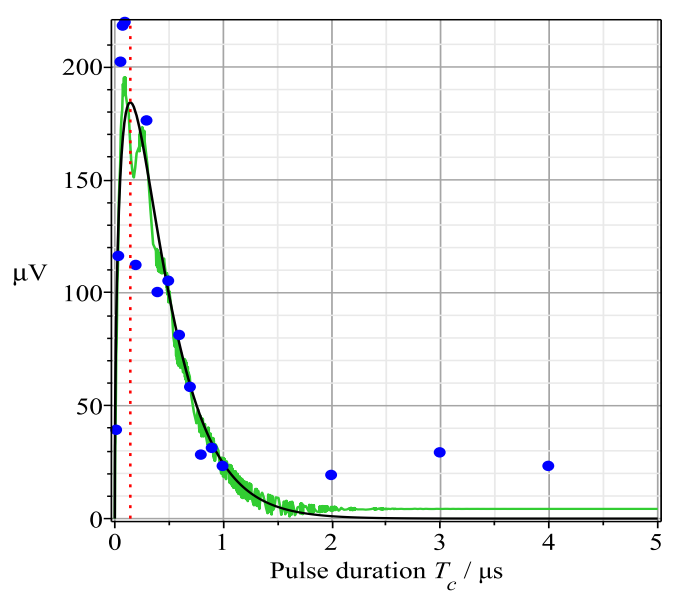

(a)

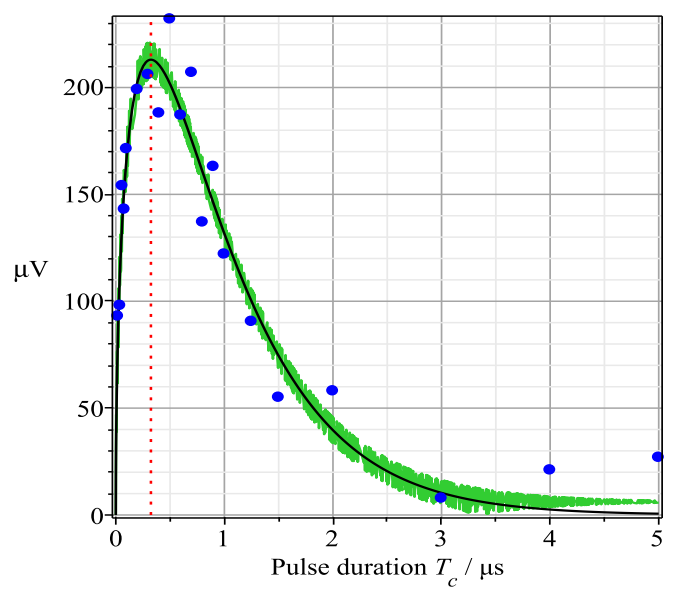

(b)

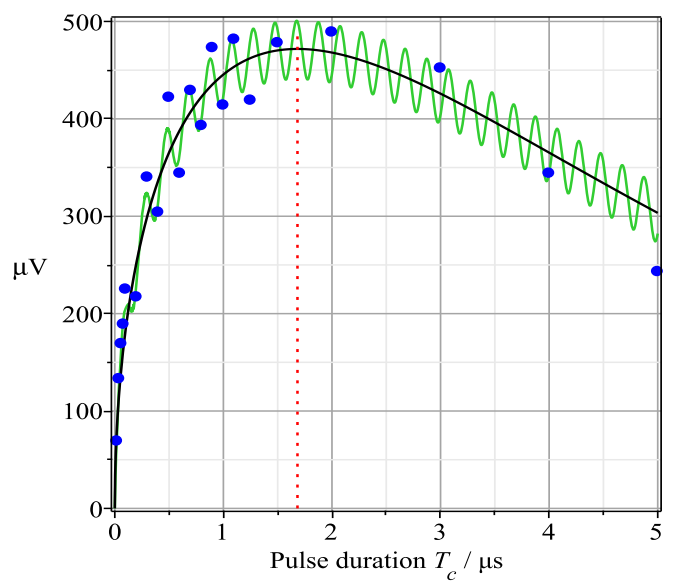

(c)

Figure 2: Comparison between experiment and models, the line position $r_{0}$ as parameter, $P=100 \mu$ bar and $\Delta \nu=1000 \mathrm{MHz}$. Blue points are experimental data and black curve is the fit using Eq. (22). The green curve is a fit where the polarization used in Eq. (18) is given by Eq. (9) rather than Eq. (17). The red vertical dotted lines indicate the positions of $T_{c, \max }$. (a)

$$
r_{0}=0.1 \text {. (b) } r_{0}=0.5 \text {. (c) } r_{0}=0.9 \text {. }
$$

a given pressure (i.e. $T_{2}$ fixed) and a given relative position $r_{0}$, we measured the FID signal for different pulse durations $T_{c}$. After a Fast Fourier Transform, the amplitude at the intermediate frequency $\omega_{I F}$ is proportional to:

$$
F\left(\omega=\omega_{I F}, T_{c}\right)=N \sqrt{\frac{T_{c}}{\Delta \omega}} e^{-\frac{T_{c}}{T_{2}}\left(1-r_{0}\right)} .
$$

These amplitudes $F\left(\omega=\omega_{I F}, T_{c}\right)$ measured for different $T_{c}$ values have been compared with two models. The simplest is given by:

$$
B P \sqrt{\frac{T_{c}}{\Delta \omega}} \exp \left[-T_{c}\left(1-r_{0}\right) 2 \pi \gamma_{\mathrm{OCS}} P\right],
$$

which is Eq. (21) reformulated with experimental parameters. In the second model, the polarization used in Eq. (18) is given by Eq. (9) rather than Eq. (17), corresponding to $\left|\xi^{(1)}\left(T_{c}\right)\right|$. The number of molecules $N$ is assumed proportional to the gas pressure $P, T_{2}=\frac{1}{2 \pi \gamma_{\mathrm{OCS}} P}$ is determined by the OCS self pressure broadening coefficient $\gamma_{\mathrm{OCS}}=4.8 \mathrm{GHz} / \mathrm{atm}$ of the $J=17 \rightarrow 16$ transition, measured in Ref. [21]. The parameter $B$ corresponds to the instrument sensitivity of a given experimental configuration. It accounts for variations in mixer conversion losses and amplifier gains. The value of $B$ is constant for a given experimental configuration. This parameter and the pressure are fitted against the experimental data sets in the second model. These two values are also used to plot the black curve corresponding to the simplest model. Figure 2 superposes the experimental data (blue points) with the fitted model of Eq. (22) (black curves) and the model corresponding to $\left|\xi^{(1)}\left(T_{c}\right)\right|$ (green curves). A good agreement between the experimental and fitted data is observed for the three different line positions $r_{0}$ at $P=100 \mu$ bar (corresponding to $T_{1} \approx T_{2}=0.3 \mu \mathrm{s}$ ) and $\Delta \nu=1000 \mathrm{MHz}$. For all experimental curves, the relative difference between measured and fitted amplitudes with the simplest model never exceeds $30 \%$. Such differences, especially around $T_{c} \approx T_{c, \max }$ for $r_{0}=0.1$ and 0.5 , are attributed to the oscillations mentioned above in Section II C and seen again on the green curves. For $r_{0}=0.9$ the oscillations are present over the entire $T_{c}$ range. Oscillations have also been discussed as "edge effects" by Park et al. [22] for short pulse durations due to the "windowing" of the perturbating electric field. This effect is reduced in our experiment by programming raised cosine edges ( $5 \mathrm{~ns}$ rise time) rather than a rectangular time window in the Arbitrary Wave Generator. The edge effects might explain the underestimated amplitude of the oscillations, given by our model (green curves), visible for $r_{0}=0.5$ at low $T_{c}$ values. The signal does not fall to zero for large $T_{c}$ values which is consistent with Fig. 1, where the first approximation coincides exactly with the numerical simulation for $\Delta \nu=1000 \mathrm{MHz}$. The amplitude of the experimental data in this region is nonetheless higher than the fitted 


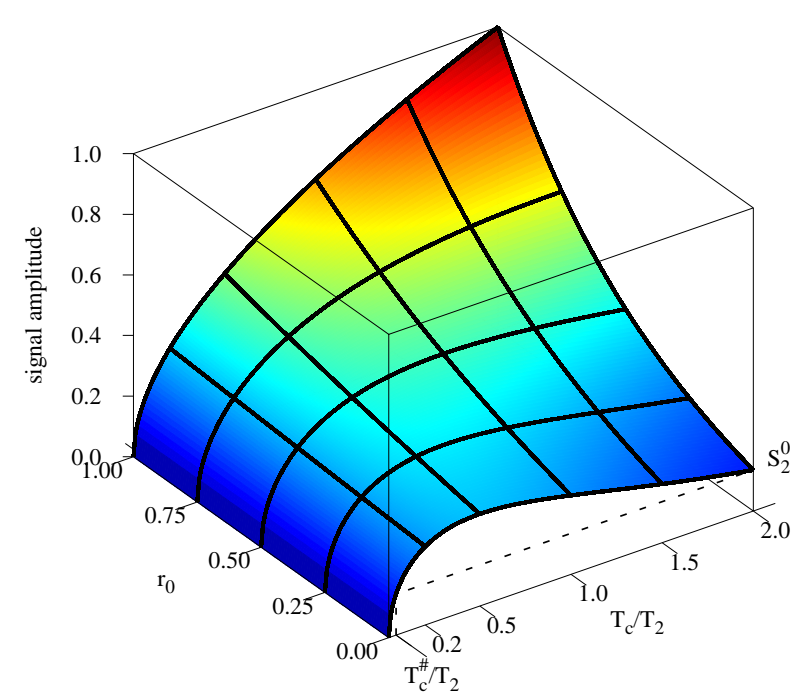

Figure 3: Three dimensional view of the amplitude of the signal $S$ in arbitrary unit from Eq. (23) versus $T_{c} / T_{2}$ and $r_{0}$ at $T_{2}$ fixed.

curve. This might suggest a shortcoming in the isolated two-level model itself. However, the results exhibited in Fig. 2 demonstrate that our simplest model with its approximations is sufficient to reproduce the behaviour of the emitted intensities in the chirped pulse experiment. This conclusion is supported by the analyses of experimental data at $P=20 \mu$ bar and $P=50 \mu$ bar which show strictly identical patterns.

\section{Discussion}

\section{Optimization of the CP parameters at a given pressure}

Eq. (20) in the case of a thermalized sample is relatively simple but depends on parameters $T_{c}, r_{0}, T_{2}$ and $N$. The signal amplitude at $t^{\prime}=0$ can be rewritten in the form of Eq. (22) to show the influence of the gas pressure or equivalently in the following form to show the influence of $T_{c} / T_{2}$ :

$$
S_{T_{c} / T_{2}}^{r_{0}} \propto \frac{1}{\sqrt{T_{2} \Delta \omega}} \sqrt{\frac{T_{c}}{T_{2}}} e^{-\frac{T_{c}}{T_{2}}\left(1-r_{0}\right)} .
$$

Experimentally, $T_{2}$ (adjusted with the gas pressure) is fixed by considerations of Fourier transform resolution. For a given $T_{2}$, the optimal pulse duration is given by $T_{c, \max }=\frac{T_{2}}{2\left(1-r_{0}\right)}$. Figure 3 is a three dimensional view of the amplitude of the signal from Eq. (23), versus $T_{c} / T_{2}$ and $r_{0}$ at $T_{2}$ fixed. The dashed line gives the signal amplitude of $S_{2}^{0}$. It shows that as long as $\frac{T_{c}}{T_{2}}<0.2$, the signal amplitude is quasi independent of the line position $r_{0}$. Inside this region, the output signal is proportional to $\sqrt{T_{c} / \Delta \omega}$ and corresponds to the approximation of a fast chirped pulse obtained by McGurk et al. [8]. Outside this region, the signal amplitude can be increased significantly but at the expense of a dependence on the line position $r_{0}$ within the pulse. However, we observe that up to $\frac{T_{c}}{T_{2}}=2$, the amplitude of the signal for $r_{0}=0$ is always greater than the amplitude for $\frac{T_{c}^{\#}}{T_{2}} \approx 0.04$. In other words, working at $T_{c} / T_{2}=2$ is more interesting than $T_{c} / T_{2} \leqslant \frac{T_{c}^{\#}}{T_{2}}$ in term of signal to noise ratio: the gain in amplitude for $r_{0}=1$ is an order of magnitude while the gain for $r_{0}=0$ is at least one.

\section{Dependence of the line strength on the line position}

Abeysekera et al. experimentally discovered the line position effect on the line intensity [7]. They considered two related experiments to mitigate the problem: in the first experiment, the frequencies are swept to higher values, whereas the frequencies are swept to lower values in the second experiment.. They proposed to average the two CP spectra to compensate for the line position effect: $S_{\text {ave }}=\left(S_{\text {up }}+S_{\text {down }}\right) / 2$. They worked with a uniform supersonic jet for which Eq. (23) applies. The signals are the same in the two experiments except that $r_{0}$ in the first one is replaced by $1-r_{0}$ in the second one. The corresponding signals are respectively $S_{\text {up }}=A e^{-\frac{T_{c}}{T_{2}}\left(1-r_{0}\right)}$ and $S_{\text {down }}=A e^{-\frac{T_{c}}{T_{2}} r_{0}}$ where $A$ is a proportionality constant. The average of the two signals,

$$
S_{\text {ave }}=A e^{-\frac{T_{c}}{2 T_{2}}} \cosh \left[\frac{T_{c}}{T_{2}}\left(r_{0}-\frac{1}{2}\right)\right],
$$

is quasi-independent of the line position $r_{0}$ if the argument of the cosh is small, i.e. for a chirp duration shorter than $T_{2}$. The series expansion of the hyperbolic cosine gives:

$$
S_{\text {ave }}=A e^{-\frac{T_{c}}{2 T_{2}}}\left(1+\frac{1}{2}\left(\frac{T_{c}}{T_{2}}\left(r_{0}-\frac{1}{2}\right)\right)^{2}+\ldots\right) .
$$

indicating that the error done is always lower than $12.5 \%$ if $T_{c} / T_{2} \leqslant 1$.

\section{CONCLUSION}

The FID signal of a two-level system interacting with a linear CP depends on the polarization at the end of the $\mathrm{CP}$. The value of this polarization, either in thermalized cases (cell or uniform supersonic jet) or non-thermalized cases (molecular beam), was approached through three consecutive analytical approximations which improve the model proposed by McGurk [8]. In particular, the last approximation neglected the oscillations in the polarization and a very simple formula for the optimal pulse duration 
(for which the modulus of the polarization is maximum) was obtained.

The simple expression between the pulse duration, the line position in the $\mathrm{CP}$ and the signal amplitude will certainly be useful in spectroscopy, where it is convenient to use slower CPs to increase the signal over a fast CP. Moreover, this relation describing the connection between the position of the lines in the $\mathrm{CP}$ and their intensity will allow for a correction of the intensities required for high resolution molecular spectroscopic studies.

\section{ACKNOWLEDGMENTS}

We would like to acknowledge the French Agence Nationale de la Recherche for their financial support of this work via funding of the project Original Submillimetre Chirped pulse instrumentation for Astrochemical Reactivity (OSCAR) under contract number ANR15-CE29-0017. The authors are also grateful for the financial support received from the Région Hauts-deFrance, the Ministère de l'Enseignement Supérieur et de la Recherche (CPER Climibio), and the European Fund for Regional Economic Development through the TERAFOOD project (INTERREG V FR-WA-VL 1.2.11).

\section{Appendix A: Doppler broadening in the Free Induction Decay}

The FID corresponds to the extinction of the source at $t=T_{c}$ and the reemission of the polarized molecules which freely evolve. We perform a change of variable in this section: $t^{\prime}=t-T_{c}$.

\section{System evolution}

Since the source is switched off, the Rabi frequency is set to zero and the optical Bloch equations in the rotating frame $[12,23]$ reduce to:

$$
\left\{\begin{array}{l}
\frac{d U}{d t}\left(t^{\prime}\right)=-\gamma_{2} U\left(t^{\prime}\right)-\delta V\left(t^{\prime}\right) \\
\frac{d V}{d t}\left(t^{\prime}\right)=-\gamma_{2} V\left(t^{\prime}\right)+\delta U\left(t^{\prime}\right) \\
\frac{d W}{d t}\left(t^{\prime}\right)=-\gamma_{1}\left(W\left(t^{\prime}\right)-W_{\mathrm{eq}}\right)
\end{array}\right.
$$

where $\delta$ is the detuning from the resonance, null if the inhomogenous broadening is negligible and $W_{\text {eq }}$ is the population difference at the thermal equilibrium. $(U, V, W)$ is the Bloch vector and the polarization is $\mathscr{P}\left(t^{\prime}\right)=$ $\left[U\left(t^{\prime}\right)-i V\left(t^{\prime}\right)\right] N \mu_{a b}$. This is a simple coupled system of differential equations. The third equation is directly integrable giving:

$$
W\left(t^{\prime}\right)=\left[W\left(T_{c}\right)-W_{\mathrm{eq}}\right] e^{-\gamma_{1} t^{\prime}}+W_{\mathrm{eq}},
$$

where $W\left(T_{c}\right)=W\left(t^{\prime}=0\right)$. The remaining part of the system is then:

$$
\left\{\begin{array}{l}
\frac{d U}{d t}\left(t^{\prime}\right)=-\gamma_{2} U\left(t^{\prime}\right)-\delta V\left(t^{\prime}\right) \\
\frac{d V}{d t}\left(t^{\prime}\right)=-\gamma_{2} V\left(t^{\prime}\right)+\delta U\left(t^{\prime}\right)
\end{array}\right.
$$

which may be diagonalizable to find eigenvalues and eigenvectors and finally the solution:

$$
\Rightarrow\left[\begin{array}{l}
U\left(t^{\prime}\right) \\
V\left(t^{\prime}\right)
\end{array}\right]=\left[\begin{array}{c}
\mathrm{e}^{-\frac{t^{\prime}}{T_{2}}}\left(\cos \left(\delta t^{\prime}\right) U_{0}-V_{0} \sin \left(\delta t^{\prime}\right)\right) \\
\mathrm{e}^{-\frac{t^{\prime}}{T_{2}}}\left(\sin \left(\delta t^{\prime}\right) U_{0}+V_{0} \cos \left(\delta t^{\prime}\right)\right)
\end{array}\right],
$$

where $U_{0}=U\left(t^{\prime}=0\right)$ and $V_{0}=V\left(t^{\prime}=0\right)$.

\section{Inhomogeneous broadening}

We must take into account all the contributions due to inhomogeneous broadening characterized by $\Delta \omega_{D}$, the Half Width at Half Maximum (HWHM) given by:

$$
\Delta \omega_{D}=\frac{\omega_{0}}{c} \sqrt{\frac{2 R T \ln 2}{M}}
$$

where $T$ is the temperature and $M$ is the molecular weight. Different molecules have different frequency resonances due to the Doppler effect and thus different detuning $\delta$. The polarization is given by the integral of Eq. (A4) over all the detunings:

$$
\left[\begin{array}{c}
U\left(t^{\prime}\right) \\
V\left(t^{\prime}\right)
\end{array}\right]=\left[\begin{array}{c}
\int_{-\infty}^{+\infty} \mathrm{e}^{-\frac{t^{\prime}}{T_{2}}}\left[\cos \left(\delta t^{\prime}\right) U_{0}-V_{0} \sin \left(\delta t^{\prime}\right)\right] \sqrt{\frac{\ln 2}{\pi}} \frac{e^{-\ln 2 \frac{\delta^{2}}{\Delta \omega_{D}^{2}}}}{\Delta \omega_{D}} d \delta \\
\int_{-\infty}^{+\infty} \mathrm{e}^{-\frac{t^{\prime}}{T_{2}}}\left[\sin \left(\delta t^{\prime}\right) U_{0}+V_{0} \cos \left(\delta t^{\prime}\right)\right] \sqrt{\frac{\ln 2}{\pi}} \frac{e^{-\ln 2 \frac{\delta^{2}}{\Delta \omega_{D}^{2}}}}{\Delta \omega_{D}} d \delta
\end{array}\right]
$$


These integrals are tabulated or can be computed with Maple [15] giving:

$$
\left[\begin{array}{c}
U\left(t^{\prime}\right) \\
V\left(t^{\prime}\right)
\end{array}\right]=\mathrm{e}^{-\frac{t^{\prime}}{T_{2}}} e^{-\frac{t^{\prime 2} \Delta \omega_{D}^{2}}{4 \ln 2}}\left[\begin{array}{c}
U_{0} \\
V_{0}
\end{array}\right]
$$

\section{Appendix B: Detection of the FID signal}

In a $\mathrm{CP}$ experiment we are interested by the electric field reemitted during the FID sequence with the polarization given by equation (A7). We calculate the electric field in the Slowly Varying Amplitude and Phase (SVAP) approximation $[8,12]$. The general forms for electric field and polarization that propagates along the $y$ axis are:

$$
\begin{aligned}
& E\left(y, t^{\prime}\right)=\frac{1}{2}\left[E_{r}\left(y, t^{\prime}\right)+i E_{i}\left(y, t^{\prime}\right)\right] e^{-i\left(\omega_{0} t^{\prime}-k y\right)}+c c, \\
& P\left(y, t^{\prime}\right)=\frac{1}{2} N \mu_{a b}\left[U\left(t^{\prime}\right)-i V\left(t^{\prime}\right)\right] e^{-i\left(\omega_{0} t^{\prime}-k y\right)}+c c .
\end{aligned}
$$

The SVAP approximation neglects the amplitude and phase variations during temporal and spatial periods:

$$
\frac{\partial E}{\partial y} \ll k E, \frac{\partial E}{\partial t^{\prime}} \ll \omega E, \frac{\partial P}{\partial t^{\prime}} \ll \omega P .
$$

If we neglect also $\frac{1}{c} \frac{\partial E}{\partial t^{\prime}}$ compared to $\frac{\partial E}{\partial y}$, we get simplified propagation equations for the real and imaginary parts:

$$
\begin{aligned}
& \frac{\partial E_{r}}{\partial y}=\frac{k}{2 \varepsilon} N \mu_{a b} V, \\
& \frac{\partial E_{i}}{\partial y}=\frac{k}{2 \varepsilon} N \mu_{a b} U,
\end{aligned}
$$

equations that we can easily integrate between $y=0$ and $y=L$ corresponding to the length of the gas cell:

$$
E\left(L, t^{\prime}\right)=\frac{k}{4 \varepsilon} N \mu_{a b} L(V+i U) e^{-i\left(\omega_{0} t^{\prime}+\Phi\right)}+c c .
$$

Finally, taking Eq. (A7) into account, we get:

$E\left(L, t^{\prime}\right)=\frac{k}{4 \varepsilon} N \mu_{a b} L e^{-\frac{t^{\prime}}{T_{2}}} e^{-\frac{t^{\prime 2} \Delta \omega_{D}^{2}}{4 \ln 2}} \sqrt{U_{0}^{2}+V_{0}^{2}} e^{-i\left(\omega_{0} t^{\prime}+\Phi-\theta\right)}+c c$,

where $\left[U_{0} ; V_{0}\right]=\left[U\left(t=T_{c}\right) ; V\left(t=T_{c}\right)\right]$.

In the experiment, we access the electric field by an heterodyne scheme tuned in the way to observe $S\left(t^{\prime}\right)=$ $A E\left(t^{\prime}\right) \cos \left(\nu_{I F} t^{\prime}+\phi\right)$ where $A$ is a constant depending on mixer efficiency, amplifier gain etc.., $\nu_{I F}$ is the intermediate frequency $\left(\nu_{I F}=\nu_{0}-\nu_{L O}\right)$ and $\phi$ the phase at the origin of time.

$$
S\left(t^{\prime}\right) \propto\left|\mathscr{P}\left(T_{c}\right)\right| e^{-\frac{t^{\prime}}{T_{2}}} e^{-\frac{t^{\prime 2} \Delta \omega_{D}^{2}}{4 \ln 2}} \cos \left(\nu_{I F} t^{\prime}+\phi\right) .
$$

[1] K. Prozument, A. P. Colombo, Y. Zhou, G. B. Park, V. S. Petrović, S. L. Coy, and R. W. Field, Phys. Rev. Lett. 107, 143001 (2011).

[2] G. G. Brown, B. C. Dian, K. O. Douglass, S. M. Geyer, S. T. Shipman, and B. H. Pate, Rev. Sci. Instrum. 79, 53103 (2008).

[3] G. B. Park, A. H. Steeves, K. K. Prozument, J. L. Neill, and R. W. Field, J. Chem. Phys. 135, 024202 (2011).

[4] A. L. Steber, B. J. Harris, J. L. Neill, and B. H. Pate, J. Mol. Spectrosc. 280, 3 (2012).

[5] F. Hindle, C. Bray, K. Hickson, D. Fontanari, M. Mouelhi, A. Cuisset, G. Mouret, and R. Bocquet, J. Infrared Millim. Terahertz Waves 39, 105 (2018).

[6] E. Gerecht, K. O. Douglass, and D. F. Plusquellic, Opt. Express 19, 8973 (2011).

[7] C. Abeysekera, L. N. Zack, G. B. Park, B. Joalland, J. M. Oldham, K. Prozument, N. M. Ariyasingha, I. R. Sims, R. W. Field, and A. G. Suits, J. Chem. Phys. 141, 214203 (2014).

[8] J. C. McGurk, T. G. Schmalz, and W. H. Flygare, J. Chem. Phys. 60, 4181 (1974).

[9] G. G. Brown, B. C. Dian, K. O. Douglass, S. M. Geyer, and B. H. Pate, J. Mol. Spectrosc. 238, 200 (2006).

[10] J. C. McGurk, T. G. Schmalz, and W. H. Flygare, Advances in chemical physics (John Wiley \& Sons, Ltd, 1974) Chap. 1, pp. 1-68.

[11] M. D. Levenson and S. S. Kano, Quantum Electronics Principles and Applications (Academic Press Inc, 1988).

[12] P. Meystre and M. Sargent III, Elements of quantum optics 3rd edition (Springer, 1999).

[13] G. B. Park and R. W. Field, J. Chem. Phys. 144 (2016).

[14] M. Abramowitz and I. A. Stegun, Handbook of Mathematical Functions (Dover Publications, 1972) equation 7.1.23.

[15] Maple 2019, Maplesoft, http://www.maplesoft.com. (2019).

[16] M. Broquier, C. Crépin, H. Dubost, and J.-P. Galaup, Chem. Phys. 341, 207 (2007).

[17] D. Bigourd, G. Mouret, A. Cuisset, F. Hindle, E. Fertein, and R. Bocquet, Opt. Commun. 281, 3111 (2008).

[18] B. Dian, G. Brown, K. Douglass, and B. Pate, Science 320, 924 (2008).

[19] J. I. Steinfeld, Molecules and Radiation (MIT Press, Cambridge, Massachusetts, 1986). 
[20] H. W. Kroto, Molecular Rotation Spectra (Dover Publications Inc. Mineola, New York, 2003).

[21] S. Matton, F. Rohart, R. Bocquet, G. Mouret, D. Bigourd, A. Cuisset, and F. Hindle, J. Mol. Spectrosc.
239, 182 (2006).

[22] G. B. Park and R. W. Field, J. Mol. Spectrosc. 312, 54 (2015).

[23] H. Mäder, H. Bomsdorf, and U. Andresen, Z. Naturforsch. 34a, 850 (1979). 
Table I: Characteristic parameters for three experimental setups. The simplified modulus of polarization (see text) and the optimal duration $T_{c, \max }$ for the maximum signal are reported. $T_{\text {rot }}$ is the molecular rotational temperature.

\begin{tabular}{|c|c|c|}
\hline Thermodynamic cond. & Thermalized case & Non thermalized case \\
\hline Experiment & Ordinary cell $\quad$ Uniform supersonic jet & Molecular beam \\
\hline$T_{\text {rot }}$ & $10-150 \mathrm{~K}$ & $5-10 \mathrm{~K}$ \\
\hline$T_{2}$ & $\approx T_{1}$ & $<T_{1}$ \\
\hline $\mathrm{W}(0)$ & $W_{\text {eq }}\left(T_{\text {rot }}\right)$ & up to $50 W_{\text {eq }}$ \\
\hline$\left|\xi_{\text {app }}^{(1)}\left(T_{c}\right)\right|$ & $\propto \sqrt{\frac{T_{c}}{\Delta \omega}}\left|W_{\mathrm{eq}}\left(T_{\mathrm{rot}}\right)\right| e^{-\frac{T_{c}}{T_{2}}\left(1-r_{0}\right)}$ & $\propto \sqrt{\frac{T_{c}}{\Delta \omega}}|W(0)|$ \\
\hline$T_{c, \max }$ & $\frac{T_{2}}{2\left(1-r_{0}\right)}$ & - \\
\hline
\end{tabular}

